\title{
Akute Otitis media: Welches Kind braucht ein Antibiotikum?
}

\section{Hier steht eine Anzeige.}

\begin{abstract}
Kinder mit stark gewölbtem Trommelfell infolge akuter Otits media profitieren am meisten von einer antimikrobiellen Behandlung, so das Ergebnis einer finnischen Studie. Hier zeigte sich auch, wie mittels Tympanometrie eine Zuordnung der Kinder möglich ist, die zunächst nur analgetisch behandelt und beobachtet werden können.
\end{abstract}

m Rahmen einer Sekundäranalyse einer randomisierten, doppelblinden, placebokontrollierten Studie haben Paula Tähtinen von der finnischen Universitätsklinik Turku und Kollegen untersucht, welche Kinder am meisten von einer antimikrobiellen Behandlung profitieren und bei welchen Patienten zunächst eine engmaschige Beobachtung unter Schmerztherapie möglich ist. Die analysierten Daten beziehen sich auf 319 Kinder zwischen 6 und 35 Monaten, die im Rahmen der Primärversorgung einer AOM über sieben Tage entweder Amoxicillin/Clavulansäure (40/5,7 mg/kg/Tag) oder Placebo erhalten hatten. Alle Kinder waren einmal gegen Haemophilus influenzae Typ b und 2,2\% von ihnen gegen Pneumokokken geimpft. Bei 91,8\% war ein pralles oder vorgewölbtes Trommelfell erkennbar, bei 76,5\% eitriges Sekret und bei 9,7\% fanden sich hämorrhagische Rötungen.

Bei 31,7\% aller Studienteilnehmer kam es zu Therapieversagen, seltener bei älteren Kindern zwischen 24 und 35 Monaten als bei jüngeren ( $20 \%$ vs. $34,4 \%$ ). Auch spitz zulaufende Tympanogramme (A- und C-Kurve) bei der Erstuntersuchung waren in der multivariaten Analyse ein Indiz für eine bessere Therapieantwort (Hazard Ratio für Therapieversagen: HR 0,53 bzw. 0,43).

In der Subgruppenanalyse zeigte sich bei Studienteilnehmern mit starker Vorwölbung des Trommelfells unter der antibiotikafreien Therapie ein nahezu doppelt so hohes Risiko für ein Therapieversagen wie bei Kindern mit moderater, geringer oder fehlender Trommelfellwölbung (HR 1,96). Dagegen war das Risiko bei schwerem Krankheitsbild gegenüber einer schwächeren Ausprägung bei antibiotischer Behandlung nicht erhöht (HR 0,51).

Die größten Gruppenunterschiede im Hinblick auf das Therapieversagen zeigten sich bei den Kindern mit starker Trommelfellwölbung (nach Antibiotikatherapie $11,1 \%$ vs. $64,1 \%$ unter Placebo). Tähtinen und Kollegen errechneten daraus eine Number needed to treat (NNT) von 1,9. Es müssten also zwei Kinder antibiotisch behandelt werden, um bei einem Kind ein Therapieversagen zu verhindern.

Fazit: Tähtinen und Kollegen gehen aufgrund ihrer Studienergebnisse davon aus, dass Kinder auch ohne schwere Symptomatik und mit nur einem schmerzenden Ohr bei starker Trommelfellvorwölbung von einer antimikrobiellen Behandlung profitieren können. Andererseits sei die Tympanometrie hilfreich, um herauszufinden, welche Kinder zunächst auf eine antibiotische Therapie verzichten könnten. Dr. Christine Starostzik

Tähtinen P A et al. Prognostic Factors for Treatment Failure in Acute Otitis Media. Pediatrics 2017; https://doi.org/10.1542/peds.2017-0072 